\title{
Chiral Light Design and Detection Inspired by Optical Antenna Theory
}

\author{
Lisa V. Poulikakos, ${ }^{\circledR}$ Prachi Thureja, Alexia Stollmann, Eva De Leo, ${ }^{\circledR}$ and David J. Norris*º
}

Optical Materials Engineering Laboratory, Department of Mechanical and Process Engineering, ETH Zurich, 8092 Zurich, Switzerland

\section{Supporting Information}

ABSTRACT: Chiral metallic nanostructures can generate evanescent fields which are more highly twisted than circularly polarized light. However, it remains unclear how best to exploit this phenomenon, hindering the optimal utilization of chiral electromagnetic fields. Here, inspired by optical antenna theory, we address this challenge by introducing chiral antenna parameters: the chirality flux efficiency and the chiral antenna aperture. These quantities, which are based on chirality conservation, quantify the generation and dissipation of chiral light. We then present a label-free experimental technique, chirality flux spectroscopy, which measures the chirality flux efficiency, providing valuable information on chiral near fields in the far field. This principle is verified theoretically and experimentally with two-dimensionally chiral coupled nanorod antennas, for which we show that chiral near and far fields are linearly dependent on the magnetoelectric polarizability. This elementary system confirms our concept to quantify chiral electromagnetic fields and paves the way toward broadly tunable chiral optical applications including ultrasensitive detection of molecular chirality or optical information storage and transfer.

KEYWORDS: Optical chirality, optical antenna, chirality flux, plasmonics, polarimetry

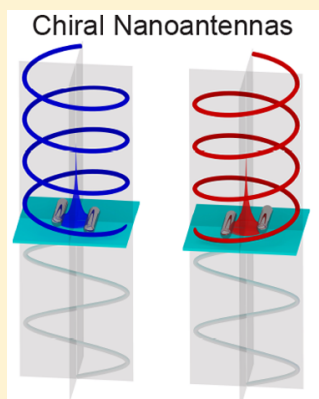
Chirality Flux Spectroscopy

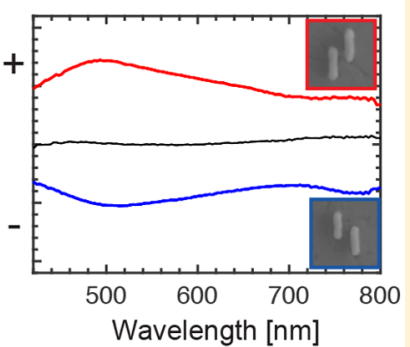

C hirality, the phenomenon of handedness, is a distinctive property of living matter. ${ }^{1}$ Molecular chirality arises due to the mirror-symmetric arrangement of otherwise identical compounds. Because the structural differences between these molecules, known as enantiomers, can impact their biochemical function, techniques for selective detection and separation of chiral molecules have been sought. ${ }^{2}$ Chiral light, such as leftand right-handed circularly polarized plane waves, offers a versatile and noninvasive means to detect molecular chirality. ${ }^{3}$ However, this approach is intrinsically limited in sensitivity due to the dimensional mismatch between molecules and the wavelength of light, ${ }^{4}$ which leads to long integration times that hinder applications. ${ }^{5}$

Recent advances in nanotechnology have enabled the fabrication of chiral metallic nanostructures ${ }^{6}$ (and achiral nanostructures excited with chiral light $^{7-9}$ ) that address the above challenge in two ways. First, their evanescent fields can exceed the chirality of circularly polarized light. ${ }^{10}$ Second, their spatial extent can better match subwavelength molecular dimensions. ${ }^{11}$ These two factors can increase detection sensitivity of chiral molecules by orders of magnitude. ${ }^{12-14}$ Thus, highly chiral evanescent fields offer a promising avenue toward the realization of ultrasensitive on-chip selection and separation of chiral molecules. ${ }^{15-20}$

However, to move beyond initial demonstrations of highly chiral light, criteria for effectively designing and tailoring nanostructures for the chirality of their fields are needed. This requires methods to characterize nanoscale chiral fields. Probing the near field directly is experimentally challenging with existing techniques. ${ }^{21}$ Ideally, we would extract information on chiral evanescent fields from far-field measurements, ${ }^{22}$ but this approach has not yet succeeded.

Optical antenna theory can help resolve this issue. Specifically, just as classical electromagnetic antennas optimize energy transfer between a localized receiver and the free radiation field, ${ }^{23-25}$ an ideal chiral nanoantenna should enhance the transfer of chiral optical information from a molecule placed in its near field to the far field (Figure 1). Thus, rather than directly probing chiral near fields, an appropriate far-field technique should be sufficient.

Here, we present a formalism for the optical characterization of chiral nanoantennas inspired by classical antenna theory. We introduce physical quantities, the chirality flux efficiency and the chiral antenna aperture, that enable the quantification of the chiral optical properties of a nanoantenna. We then develop a label-free experimental technique, chirality flux spectroscopy, to measure the chirality flux efficiency and verify our results with both theory and experiment for coupled nanorod dimers with two-dimensional (2D) chirality. We find that the chiral fields of the system are driven by the magnetoelectric polarizability,

Received: January 8, 2018

Revised: March 12, 2018

Published: March 13, 2018 


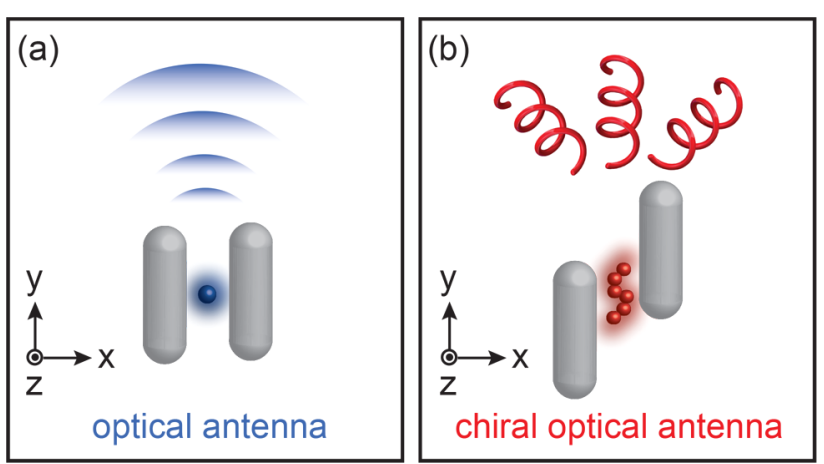

Figure 1. Conceptual illustration of (a) an optical antenna where energy transfer to and from a receiver is enhanced and (b) a chiral optical antenna where the receiver is a chiral molecule and the transfer of chiral optical information to and from the far field is enhanced.

exhibiting a linear relationship between chirality flux efficiency and the chiral near-field coupling of the two nanorods in the studied regime. Thus, our method provides vital information on chiral near fields that can be accessed in the far field, enabling rational nanoantenna design based on chiral optical fields and the optimal utilization of chiral light in specifically tailored applications.

The handedness of chiral electromagnetic plane waves is determined by the rotation direction around the propagation axis. Locally, this is quantified by the optical chirality density, ${ }^{26}$ which in its time-averaged form (denoted with an overbar) is

$$
\bar{\chi}=-\frac{\omega}{2} \operatorname{Im}\left(\mathcal{D}^{*} \cdot \mathcal{B}\right)
$$

where $\omega$ is the angular frequency and $\mathcal{D}$ and $\mathcal{B}$ are the complex amplitudes for the electric displacement and magnetic fields, respectively, with the asterisk indicating the complex conjugate. $\bar{\chi}$ is a chiral analogue to the local density of optical states. $^{27}$ For a single plane wave, $\bar{\chi}$ reaches its extrema for circularly polarized light $(\mathrm{CPL}) .{ }^{28}$ This limit $\left(\bar{\chi}_{\mathrm{CPL}}\right)$ can be exceeded by evanescent waves, due to their theoretically unlimited in-plane spatial frequencies. ${ }^{29}$ Specifically, a $z$ decaying evanescent wave with in-plane wavevector components $k_{x}$ and $k_{y}$ reaches its maximum optical chirality density at

$$
\bar{\chi}_{\text {max }, \mathrm{ev}}= \pm \varepsilon_{0}\left|\mathcal{E}_{z}\right|\left(\left|\mathcal{E}_{x}\right| k_{y}+\left|\mathcal{E}_{y}\right| k_{x}\right)
$$

where $\varepsilon_{0}$ is the vacuum permittivity and $\mathcal{E}$ is the complex electric-field amplitude with Cartesian components $\mathcal{E}_{x}, \mathcal{E}_{y}$, and $\mathcal{E}_{z}$ (see the Supporting Information). Chiral metallic nanostructures, which are effective generators of chiral evanescent fields, ${ }^{6}$ therefore offer an accessible route toward chiral sensing.

Previously, these systems have been characterized using spectroscopic techniques ${ }^{21}$ designed to evaluate biomolecular structure. ${ }^{30}$ Most notably, circular dichroism (CD) spectrosco$\mathrm{py}^{3,4}$ measures the differential extinction between illumination with left- and right-handed circularly polarized light. In addition to the conventional extinction approach, variations of $\mathrm{CD}$ have been developed on the basis of nonlinear optical response, ${ }^{31}$ strong optical scattering, ${ }^{32}$ and fluorescence. ${ }^{33}$ These have been successfully applied to chiral metallic nanostructures. ${ }^{34-40}$ Compared to molecules, plasmonic resonances can exhibit CD signal amplitudes that are orders of magnitude higher at ultraviolet, visible, and near-infrared frequencies. ${ }^{41-45}$ Indeed, geometries to maximize this effect have been proposed. ${ }^{46}$ Moreover, these signals can be induced or altered by ultravioletresonant molecules. ${ }^{47}$ However, while these spectra provide valuable structural information, their relation to chiral evanescent fields is unclear. 22

Instead of alternating the handedness of circularly polarized illumination (as in CD spectroscopy), one can alternatively evaluate the degree of circular polarization of the fields that are emitted or scattered by a chiral sample. Circularly polarized luminescence $^{48}$ exploits this approach for chiral emitters. Moreover, it has been applied to chiral metallic nanostructures by functionalizing their surface with achiral fluorophores. 49,50

Table 1. Comparison of Optical Antenna Parameters (left) and Chiral Optical Antenna Parameters (right) Based on Their Governing Conservation Laws, Where $\omega$ is the Angular Frequency and $\mathbf{n}$ Is the Normal Vector ${ }^{a}$

\begin{tabular}{c|c}
\hline Optical Antenna Parameters & Chiral Optical Antenna Parameters \\
\hline$\underbrace{2 \omega \int_{V} \operatorname{Im}\left(w_{e}-w_{m}\right) \mathrm{d}^{3} \mathrm{x}}_{P_{\text {diss }}}=\underbrace{\int_{S} \operatorname{Re}(\boldsymbol{S} \cdot \mathbf{n}) \mathrm{d}^{2} \mathrm{x}}_{P_{\text {scat }}}$ & $\underbrace{2 \omega \int_{\mathrm{V}} \operatorname{Im}\left(\chi_{e}-\chi_{m}\right) \mathrm{d}^{3} \mathrm{x}}_{\overline{\mathrm{V}}}=\underbrace{\int_{S} \operatorname{Re}(\mathcal{F} \cdot \mathbf{n}) \mathrm{d}^{2} \mathrm{x}}_{\overline{\mathcal{F}}}$ \\
\hline Antenna Efficiency & Chirality Flux Efficiency \\
\hline$:=\frac{P_{\text {scat }}}{P_{\text {ext }}}[-]$ & $\eta_{\overline{\mathcal{F}}}:=\frac{c}{\omega P_{\text {tot }}} \overline{\mathcal{F}}[-]$ \\
Antenna Aperture & Chiral Antenna Aperture \\
$\sigma:=\frac{P_{\text {diss }}}{I_{\text {inc }}}\left[\mathrm{m}^{2}\right]$ & $\sigma_{\bar{X}}:=\frac{\bar{X}}{p_{\text {inc }}}\left[\mathrm{m}^{2}\right]$
\end{tabular}

${ }^{a}$ In the left column, $w_{\mathrm{e}}$ and $w_{\mathrm{m}}$ are the complex harmonic electric and magnetic energy densities, ${ }^{22} \operatorname{Im}\left(w_{\mathrm{e}}-w_{\mathrm{m}}\right)=\frac{1}{4} \operatorname{Im}\left(\mathcal{E} \cdot \mathcal{D}^{*}-\mathcal{B} \cdot \mathcal{H}^{*}\right), \mathcal{S}$ is the Poynting vector, $P_{\text {diss }}$ is the dissipated power, $P_{\text {scat }}$ is the scattered power, $P_{\text {ext }}=P_{\text {diss }}+P_{\text {scat }}$ is the extinction power, and $I_{\text {inc }}$ is the incident light intensity. In the right column, $\chi_{\mathrm{e}}$ and $\chi_{\mathrm{m}}$ are the complex harmonic electric and magnetic optical chirality $\operatorname{densities}{ }^{22} \operatorname{Im}\left(\chi_{\mathrm{e}}-\chi_{\mathrm{m}}\right)=$ $\frac{1}{8}\left[-\nabla \epsilon^{\prime} \cdot \operatorname{Im}\left(\mathcal{E} \times \mathcal{E}^{*}\right)-\nabla \mu^{\prime} \cdot \operatorname{Im}\left(\mathcal{H} \times \mathcal{H}^{*}\right)\right]+\frac{1}{4} \omega\left(\epsilon^{\prime} \mu^{\prime \prime}+\epsilon^{\prime \prime} \mu^{\prime}\right) \operatorname{Im}\left(\mathcal{E}^{*} \cdot \mathcal{H}\right), \mathcal{F}$ is the optical chirality flux, $c$ is the speed of light, $P_{\text {tot }}$ is the total power of the outgoing light, and $p_{\text {inc }}$ is the incident power density. 
Although this strategy can probe chiral near fields, two disadvantages remain: (i) the resulting spectra are convoluted with fluorophore emission ${ }^{51}$ and (ii) the metal surface must be decorated with fluorescent labels, which can hinder subsequent interactions between the near field and a chiral analyte of interest.

We now introduce an approach that enables the label-free detection of chiral optical fields. By extending optical antenna theory, we can theoretically and experimentally quantify the generation and dissipation of chiral light in terms of parameters that directly relate to the chiral evanescent fields. As optical antenna parameters are derived from energy conservation, ${ }^{29}$ we base our technique on the analogous conservation law of optical chirality in lossy, dispersive media. ${ }^{22}$ This requires optical chirality dissipation to balance with the optical chirality flux (see Table 1). Thus, by detecting the degree of circular polarization of the scattered light in the far field, the optical chirality flux can be determined. This then provides information about the chiral near field (see the Supporting Information).

We define two antenna parameters to characterize chiral nanoantennas, the chirality flux efficiency $\eta_{\overline{\mathcal{F}}}[-]$ and the chiral antenna aperture $\sigma_{\bar{X}}\left[\mathrm{~m}^{2}\right]$ (see the Supporting Information for further details)

$$
\begin{aligned}
\eta_{\overline{\mathcal{F}}}:=\frac{c}{\omega P_{\text {tot }}} \overline{\mathcal{F}} \\
\sigma_{\bar{X}}:=\frac{\bar{X}}{p_{\text {inc }}}
\end{aligned}
$$

where $c$ is the speed of light in vacuum and

$$
\overline{\mathcal{F}}=\int_{S} \operatorname{Re}(\mathcal{F} \cdot \boldsymbol{n}) \mathrm{d}^{2} x
$$

is the integral of the optical chirality flux, $\mathcal{F}$, through the surface $S{ }^{22}$ with

$$
\mathcal{F}=\frac{1}{4}\left[\mathcal{E} \times\left(\nabla \times \mathcal{H}^{*}\right)-\mathcal{H}^{*} \times(\nabla \times \mathcal{E})\right]
$$

$\mathcal{H}$ is the complex amplitude of the $\mathbf{H}$ field, and $P_{\text {tot }}$ is the total power of the outgoing light. Further,

$$
\bar{X}=2 \omega \int_{V} \operatorname{Im}\left(\chi_{\mathrm{e}}-\chi_{\mathrm{m}}\right) \mathrm{d}^{3} x
$$

is the volume-integrated optical chirality dissipation ${ }^{22,52}$ with

$$
\begin{aligned}
& \operatorname{Im}\left(\chi_{\mathrm{e}}-\chi_{\mathrm{m}}\right)= \\
& \frac{1}{8}\left[-\nabla \epsilon^{\prime} \cdot \operatorname{Im}\left(\mathcal{E} \times \mathcal{E}^{*}\right)-\nabla \mu^{\prime} \cdot \operatorname{Im}\left(\mathcal{H} \times \mathcal{H}^{*}\right)\right] \\
& \quad+\frac{1}{4} \omega\left(\epsilon^{\prime} \mu^{\prime \prime}+\epsilon^{\prime \prime} \mu^{\prime}\right) \operatorname{Im}\left(\mathcal{E}^{*} \cdot \mathcal{H}\right)
\end{aligned}
$$

with $\epsilon=\epsilon^{\prime}+\mathrm{i} \epsilon^{\prime \prime}$ as the complex permittivity, $\mu=\mu^{\prime}+\mathrm{i} \mu^{\prime \prime}$ as the complex permeability, and $\chi_{\mathrm{e}}, \chi_{\mathrm{m}}$ as the complex harmonic electric and magnetic optical chirality densities. ${ }^{22}$ Finally, $p_{\text {inc }}$ is the incident power density.

While the conventional antenna efficiency quantifies radiative power generation, ${ }^{29}$ eq 3 describes the ability of the antenna to scatter chiral optical fields. Similarly, as the conventional antenna aperture describes power dissipation, related to the absorption cross section, ${ }^{29}$ eq 4 describes the dissipation of chiral optical fields which, for small metallic nanostructures with negligible damping and retardation, can be brought into connection with the near-field optical chirality density. ${ }^{22}$ The comparison to optical antenna parameters is summarized in Table 1.

We now apply the chiral antenna parameters in eqs 3 and 4 to an analytical model of the $2 \mathrm{D}$ chiral metallic nanoantenna studied here. In the quasi-static electric-dipole limit, this system is described by the dipole moment $\mathbf{p}=\alpha_{\mathrm{e}} \mathcal{E}+\mathrm{i} \alpha_{\mathrm{c}} \mathcal{H}{ }^{53,54}$ The electric polarizability is written as $\alpha_{\mathrm{e}}$ and the chiral optical properties are attributed to $\alpha_{\mathcal{c}}$ the coupled magnetoelectric polarizability. ${ }^{55}$ Inserting this model into eqs 1,3 , and 4 , we obtain (see the Supporting Information)

$$
\begin{aligned}
& \eta_{\overline{\mathcal{F}}, \mathrm{d}}=\operatorname{Re}\left(\alpha_{\mathrm{c}}\right) \frac{3}{8 \mu_{0}} \frac{\left|\mathcal{E}_{0}\right|^{2}}{P_{\text {tot }}} \frac{1}{\sqrt{r^{2}+\zeta^{2}}} \\
& \sigma_{\bar{X}, \mathrm{~d}}=-\operatorname{Re}\left(\alpha_{\mathrm{c}}\right) \frac{\epsilon^{\prime \prime} \mu^{\prime}}{2 \mu_{0}^{2}} \frac{\left(\epsilon^{\prime}-\epsilon_{0}\right)}{\left(\epsilon^{\prime}-\epsilon_{0}\right)^{2}+\epsilon^{\prime 2}} \frac{k^{2}\left|\mathcal{E}_{0}\right|^{2}}{p_{\text {inc }}} \\
& \bar{\chi}_{\mathrm{d}}=-\operatorname{Re}\left(\alpha_{\mathrm{c}}\right) \frac{k^{2}\left|\mathcal{E}_{0}\right|^{2}}{8 \pi r^{3} \omega \mu_{0}}
\end{aligned}
$$

where $r$ and $\zeta$ are the radial and longitudinal cylindrical coordinates, $\mu_{0}$ is the vacuum permeability, and $k$ is the wavevector magnitude. Equations 9-11 have several important physical implications. First, the resonant properties of $\eta_{\overline{\mathcal{F}}, \mathrm{d}}$, $\sigma_{\bar{X}, \mathrm{~d}}$, and $\bar{\chi}_{\mathrm{d}}$ are linearly dependent on $\operatorname{Re}\left(\alpha_{\mathrm{c}}\right)$. Due to this proportionality, the magnetoelectric polarizability $\alpha_{\mathrm{c}}$ directly determines the strength of the chiral optical fields generated. Second, in the dipole limit, the resonance of $\eta_{\overline{\mathcal{F}}, \mathrm{d}}$, which is measurable in the far field, matches the resonance of $\bar{\chi}_{\mathrm{d}}$, which describes the local near fields. Third, $\sigma_{\bar{X}, \mathrm{~d}}$ quantifies the ability of the dipolar nanoantenna to dissipate optical chirality. As predicted by the conservation law of optical chirality (see the Supporting Information), the resonance with maximum $\sigma_{\bar{X}, \mathrm{~d}}$ matches that of $\eta_{\overline{\mathcal{F}}, \mathrm{d}}$, which is measurable in the far field.

We verify our theoretical findings experimentally with $2 \mathrm{D}$ chiral nanorod dimers (NRDs) whose dominant properties are dipolar in the studied regime. Figure 2a,b shows scanningelectron micrographs of right- (R) and left-handed (L) NRDs, arranged periodically with an edge-to-edge distance of $p=600$ $\mathrm{nm}\left(>6 \times 10^{4}\right.$ unit cells $)$. The rod dimensions are $70 \mathrm{~nm} \times 230$ $\mathrm{nm} \times 50 \mathrm{~nm}$ in $x, y$, and $z$ (with axes as in Figure 1) to generate sufficient antenna-scattered light. $2 \mathrm{D}$ chirality arises due to a $\pm 140 \mathrm{~nm}$ vertical and $60 \mathrm{~nm}$ horizontal shift $(h)$ between the nanorods in each pair (see the Supporting Information). Nonlinear, multipolar effects arising at small $h$ are negligible. ${ }^{56}$ The chiral coupling between two metallic nanorods induces a magnetoelectric polarizability, ${ }^{57}$ and the physical behavior of this system can thus be modeled analytically as a single $2 \mathrm{D}$ chiral dipole.

The antennas were fabricated by template stripping ${ }^{58}$ thermally evaporated silver from a structured silicon template onto a glass coverslip, after selective removal of the flat silver deposited around the nanorods ${ }^{59}$ (see the Supporting Information). An example of the optical extinction spectrum for a specific R-NRD array $(h=60 \mathrm{~nm})$ can be seen on a linear scale in Figure 2c (see the Supporting Information) for $x$ polarized incident light (axes as in Figure 1). The inset of Figure $2 \mathrm{c}$ indicates one R-NRD unit cell with an asterisk marking the center point at $(0,0,0)$. The spectrum is broad because in this demonstration we are exploiting a higher-order 


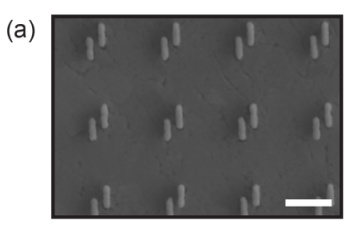

(d)

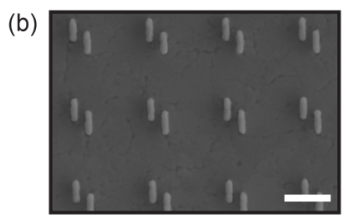

(c)
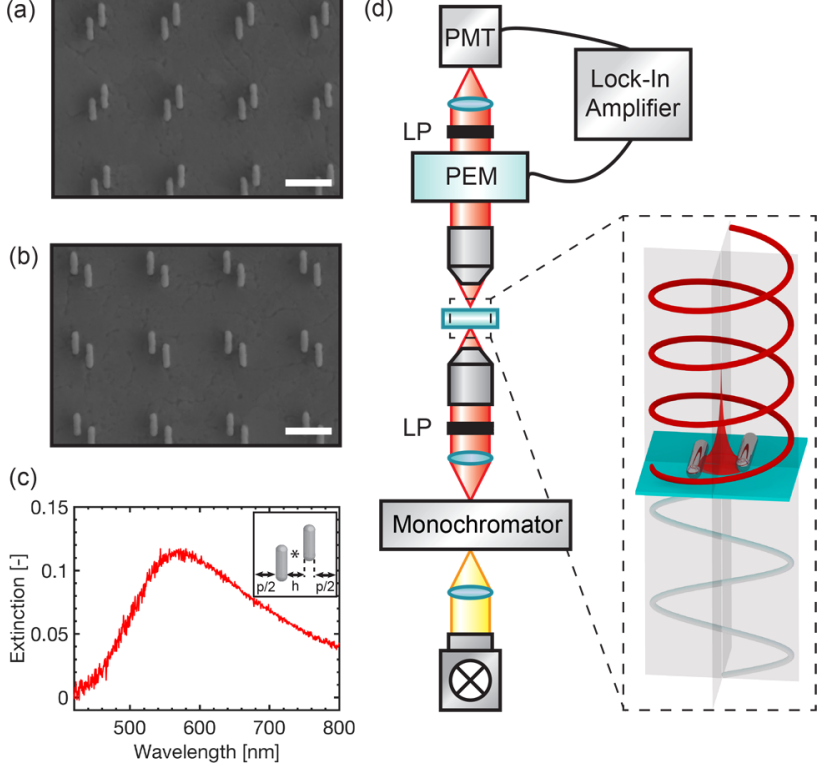

Figure 2. (a, b) Scanning-electron micrographs of (a) R-NRD and (b) L-NRD arrays with a horizontal shift of $h=60 \mathrm{~nm}$ and edge-to-edge distance of $p=600 \mathrm{~nm}$ (between outer NRD edges). The scale bars are $500 \mathrm{~nm}$. (c) Extinction spectrum of the R-NRD array, $h=60 \mathrm{~nm}$. Inset: schematic of an R-NRD unit cell where $*$ denotes the center point $(0,0,0)$. (d) The experimental setup for measuring chirality flux efficiency $\left(\eta_{\overline{\mathcal{F}}}\right.$, eq 3$)$. Inset: Schematic of sample excitation with achiral, linearly polarized light. While generated chiral evanescent fields decay, $\eta_{\overline{\mathcal{F}}}$ is detected in the far field by analysis of the scattered degree of circular polarization. For this, the light is sent through a photoelastic modulator (PEM) and subsequent linear polarizer (LP). The optical signal is detected by a photomultiplier tube (PMT) and filtered with a lock-in amplifier at the PEM modulation frequency.

resonance of the system to increase the scattering signal from the NRDs at visible frequencies.

The principle of chirality flux spectroscopy, our experimental technique to measure $\eta_{\overline{\mathcal{F}}}$, is shown schematically in Figure $2 \mathrm{~d}$. Light from a broadband illumination source (100 W halogen lamp) is passed through a monochromator and a linear polarizer (LP) before it is weakly focused [20X, 0.45 numerical aperture (NA) objective] onto the sample. The outgoing signal is collected with a $10 \times, 0.3 \mathrm{NA}$ objective. As this study focuses on single NRD coupling, low NA collection avoids lattice effects arising at higher angles. ${ }^{50}$

Next, a photoelastic modulator (PEM, Hinds Instruments I/ FS50) applies quarter-wave retardation to the collected light at $50 \mathrm{kHz}$, which is subsequently analyzed by another LP. The excitation and collection LPs are aligned precisely $\pm 45^{\circ}$ to the PEM optical axis as even minor misalignments can lead to linear birefringence artifacts. A photomultiplier tube (PMT, Hamamatsu R928) then detects the optical signal. The output is fed into a lock-in amplifier (Zurich Instruments, HF2) connected to the electronic signal from the PEM.

The PEM transforms R- or L-CPL, scattered from the sample, into linear polarization at $\pm 45^{\circ}$ to the PEM axis, leading to $50 \mathrm{kHz}$ modulation after the analyzing LP. In contrast, linear polarization, predominantly from the excitation beam, is transformed into circular polarization, which is then modulated at $100 \mathrm{kHz}$. This polarization-selective modulation enables the isolated detection of circularly polarized scattered fields, directly proportional to $\eta_{\overline{\mathcal{F}}}$, in a bright-field measurement.

The inset of Figure $2 \mathrm{~d}$ illustrates how the measurement of $\eta_{\overline{\mathcal{F}}}$ quantifies the ability of a nanoantenna to generate chiral optical fields. The excitation with achiral, linearly polarized light is key to this method, as $\eta_{\overline{\mathcal{F}}}$ becomes meaningful for a system where the sample is the only source of chiral optical fields. In this case, a relationship between chiral near and far fields can be established, because the chiral light scattered into the far field is a direct consequence of the rapidly decaying chiral evanescent fields. Specifically, $\eta_{\overline{\mathcal{F}}}$ selectively detects the excess of one handedness of chiral light scattered by the sample, modulated at $50 \mathrm{kHz}$, while equal amounts of L- and R-CPL recombine additively to linear polarization, modulated at $100 \mathrm{kHz}$.

We emphasize that this near-field information is not revealed with CD spectroscopy, in which circularly polarized excitation is used. In that case, the chiral sample's preferential extinction of one handedness of CPL results in differential amplitudes of L- and R-CPL generating an outgoing elliptical beam (see the Supporting Information). ${ }^{4}$ Thus, a CD spectrum describes how the sample alters incident chiral light, providing information on chiral geometry, ${ }^{32}$ but does not reveal its ability to generate chiral fields.

Furthermore, in optical rotatory dispersion (ORD), a spectroscopic technique which is Kramers-Kronig-related to $\mathrm{CD},{ }^{57}$ a sample is excited with linearly polarized light and the rotation plane of the outgoing elliptical beam is recorded. This effect is related to the net phase retardation experienced by Land R-CPL when traversing a circularly birefringent medium. ${ }^{30}$ Thus, ORD is clearly distinct from chirality flux spectroscopy, where polarimetric analysis directly detects an excess of L- or RCPL in the scattered fields.

We note that plasmonic quarter-wave plates have been developed to generate CPL upon linearly polarized excitation. $^{60,61}$ These systems rely on linear birefringence, where two orthogonal components of the incident linearly polarized beam obtain a net phase retardation. ${ }^{30}$ Linear birefringence does not occur for our measurement of $\eta_{\overline{\mathcal{F}}}$, as our $x$-polarized excitation (axes as in Figure 1) experiences a single refractive index. Thus, the chiral fields detected here result from the nanoantenna's near-field chiral coupling or, in other words, intrinsic rather than extrinsic chirality. ${ }^{62,63}$

Figure 3a shows experimental $\eta_{\overline{\mathcal{F}}}$ spectra for R-NRDs (red) and L-NRDs (blue), respectively ( $h=60 \mathrm{~nm}$, achiral NRDs in black, see the Supporting Information). The NRD handedness determines the sign of the generated chiral optical fields, leading to mirror-symmetric spectra. These results are well reproduced numerically in Figure $3 b$, where finite-element simulations (COMSOL Multiphysics 5.2) were conducted with $x$-polarized plane-wave excitation with periodic boundary conditions (see the Supporting Information). Amplitude differences between experimental (Figure 3a) and simulated data (Figure $3 \mathrm{~b}$ ) can be attributed to the higher collection angle in experiment $(\mathrm{NA}=0.3$, see the Supporting Information). The feature at $600 \mathrm{~nm}$ (marked with $*$ ) in Figure $3 \mathrm{~b}$ arises from the surface lattice resonance of the periodic array (see the Supporting Information). It is not seen in experiment, presumably due to damping effects and fabrication imperfections. ${ }^{50}$ By setting the edge-to-edge NRD distance to $600 \mathrm{~nm}$, we sufficiently separate lattice and single-nanoantenna resonances to distinguish their effects. 

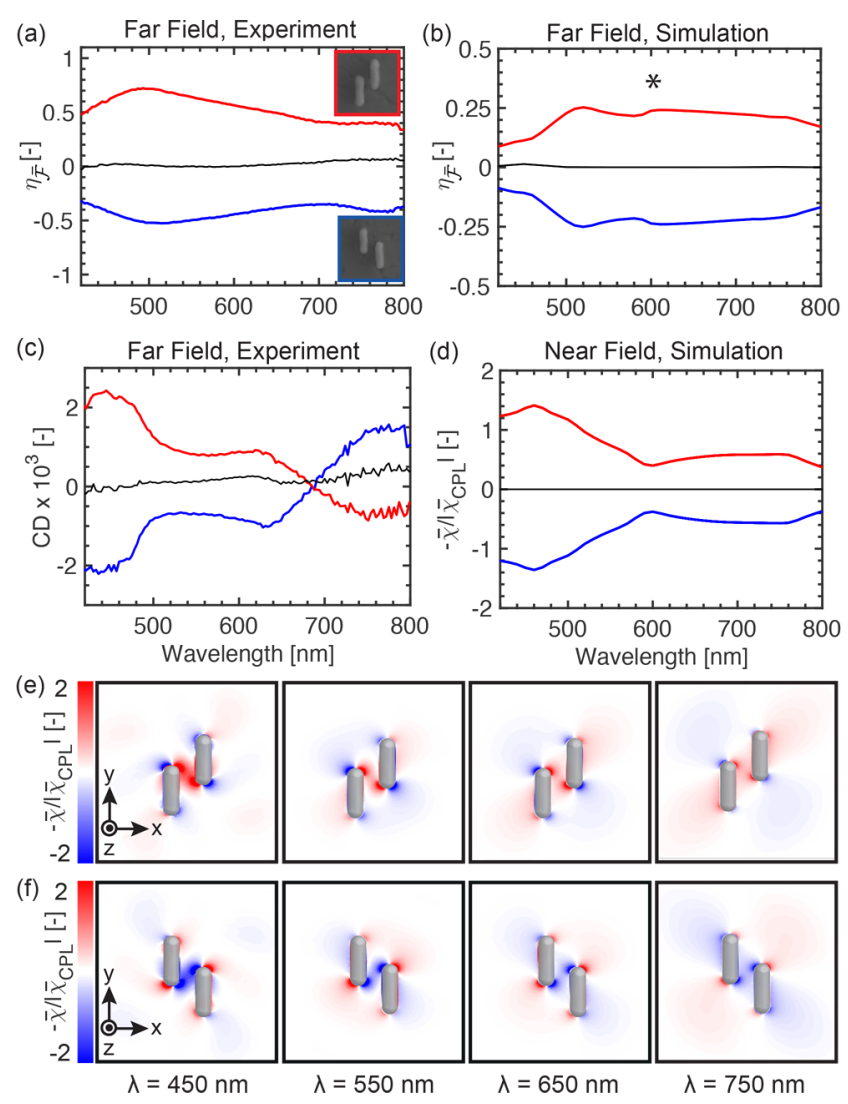

Figure 3. Experimental and simulated analysis of chiral optical fields for R-NRDs (red), L-NRDs (blue), and achiral NRDs (black) with $h=$ $60 \mathrm{~nm}$. (a) Experimental chirality flux efficiency $\left(\eta_{\overline{\mathcal{F}}}\right)$ spectra. NRDs of opposite handedness show mirror-symmetric $\eta_{\overline{\mathcal{F}}}$ spectra, while achiral NRDs show negligible $\eta_{\overline{\mathcal{F}}}$. (b) Simulated $\eta_{\overline{\mathcal{F}}}$ spectra, in good agreement with the experimental data in part a. Surface lattice effects (marked with $*$ ) are not seen in experiment due to damping and fabrication imperfections. (c) Experimental circular dichroism (CD) spectra, which differ from $\eta_{\overline{\mathcal{F}}}$ in magnitude, sign, and resonant wavelengths. (d) Simulated optical chirality enhancement $\left(\bar{\chi} / \bar{\chi}_{\mathrm{CPL}} \mid\right)$ spectra, evaluated at $(0,0,0)$, the center point between nanorods (see Figure $2 \mathrm{c}$ ). The spectral properties match those of $\eta_{\overline{\mathcal{F}}}$, where the spectral shift and broadening in parts $\mathrm{a}$ and $\mathrm{b}$ are explained by damping, retardation, ensemble effects, and low NA collection. (e, f) Simulated near-field maps $(z=0$ plane, NRD center $)$ of $\bar{\chi} / \bar{\chi}_{\mathrm{CPL}} \mathrm{L}$ for (e) R-NRDs and (f) L-NRDs at various wavelengths $(\lambda)$, which further illustrate the trends in part $\mathrm{d}$.

For comparison, the experimental setup in Figure $2 \mathrm{~d}$ was modified to perform CD measurements (see the Supporting Information). The resulting spectra in Figure $3 c$ show that, while both techniques yield characteristic mirror-symmetric trajectories for R- and L-NRDs, they clearly differ in magnitude, sign, and resonant wavelengths. The discrepancy between Figure $3 \mathrm{a}$ and $\mathrm{c}$ verifies that $\eta_{\overline{\mathcal{F}}}$ and $\mathrm{CD}$ provide different physical information.

In Figure $3 \mathrm{~d}$, the numerical optical chirality enhancement $(\bar{\chi})$ $\left.\left|\bar{\chi}_{\mathrm{CPL}}\right|\right)$ of the NRD evanescent fields is plotted versus wavelength. This parameter was evaluated at the NRD center $(0,0,0)$ (marked with $*$ in Figure $2 \mathrm{c}$ ). For both $\mathrm{R}$ - and LNRDs, $\bar{\chi} / \bar{\chi}_{\mathrm{CPL}} \mid$ retains the same sign at all wavelengths, with its maximum magnitude at $470 \mathrm{~nm}$. This trend is further confirmed in numerical colormaps of $\bar{\chi} / \bar{\chi}_{\mathrm{CPL}} \mathrm{L}(z=0$ plane at NRD center) shown in Figure $3 \mathrm{e}, \mathrm{f}$ for various wavelengths $(\lambda)$.
Here, the handedness of $\bar{\chi} / \bar{\chi}_{\mathrm{CPL}} \mid$ arises due to NRD near-field coupling and changes sign depending on the vertical shift (positive or negative) between the nanorods. This does not occur for the achiral NRDs, where no such excess is present (see the Supporting Information).

Comparing these results to Figure $3 a, b$, we find good agreement in the relative magnitude and sign of $\bar{\chi} / \bar{\chi}_{\mathrm{CPL}} \mid$ and $\eta_{\overline{\mathcal{F}}}$. This confirms the relationship between $\eta_{\overline{\mathcal{F}}}$ and chiral evanescent fields. However, differences between $\bar{\chi} / \bar{\chi}_{\mathrm{CPL}} \mathrm{L}$ and $\eta_{\overline{\mathcal{F}}}$ are also observed. These include (i) a $\sim 50 \mathrm{~nm}$ spectral shift and (ii) a broadening between near- and far-field spectra. Indeed, as with the analogous energy parameters, (i) and (ii) are plausible effects arising due to damping and retardation beyond the dipole limit, ${ }^{64}$ as well as ensemble effects and low NA collection. ${ }^{65}$ In contrast, no such connection can be drawn between $\bar{\chi} / \bar{\chi}_{\mathrm{CPL}} \mid$ and the $\mathrm{CD}$ spectrum (Figure $3 \mathrm{c}$ ).

As derived from our theoretical model, Figure 4 demonstrates how the magnetoelectric polarizability $\alpha_{c}$ drives the NRD's chiral fields, which arise from plasmonic near-field coupling between two nanorods. Experimental $\eta_{\overline{\mathcal{F}}}$ spectra are shown in Figure $4 \mathrm{a}$ where the horizontal shift $h$ varies from 60 to $200 \mathrm{~nm}$. We note that different lattice densities were accounted for by our normalization procedure (see the Supporting Information). R- (red) and L-NRDs (blue) maintain mirror-symmetric $\eta_{\overline{\mathcal{F}}}$ spectra for all $h$. As $h$ increases and the nanorod near-field coupling decreases, the magnitude of $\eta_{\overline{\mathcal{F}}}$ also decreases. Specifically, Figure $4 \mathrm{~b}$ reveals a linear trend for the extrema of $\eta_{\overline{\mathcal{F}}}$ vs $h$. This was predicted by our analytical model (eq 9), which states that $\eta_{\overline{\mathcal{F}}}$ is dependent on the magnetoelectric polarizability $\operatorname{Re}\left(\alpha_{\mathrm{c}}\right)$ in the $2 \mathrm{D}$ chiral dipole limit. In our system, $\operatorname{Re}\left(\alpha_{\mathrm{c}}\right)$ is linearly related to $h$, because the magnetoelectric polarizability is induced by chiral near-field coupling between nanorods. The relationship between $\operatorname{Re}\left(\alpha_{\mathrm{c}}\right)$ and $h$ can be understood by comparison to magnetostatics: ${ }^{66}$ due to plasmon hybridization, ${ }^{67}$ the plasmon-induced surface charges cause oscillating currents across the chiral NRD which induce the magnetoelectric dipolar component. Just as the current density along a circular loop is inversely proportional to the loop radius, by increasing $h$ in our chiral NRD system, the effective NRD radius increases and we expect a decrease in the chiral optical fields, which is well-approximated as linear in the studied $h$-regime.

Further, our analytical model predicts that the chiral near fields also depend linearly on $\operatorname{Re}\left(\alpha_{\mathrm{c}}\right)$ (eq 11). Indeed, Figure 4c confirms this numerically for $\bar{\chi} / \bar{\chi}_{\mathrm{CPL}} \mid$ evaluated at the NRD center $(0,0,0)$ (marked with $*$ in Figure $2 c$ ). The decrease in near-field optical chirality with increasing $h$ is further illustrated with numerical colormaps of $\bar{\chi} / \bar{\chi}_{\mathrm{CPL}} \mid$ for varying $h$ in Figure $4 \mathrm{~d}$. These results allow two important conclusions: the predominant optical properties of the studied NRD system are dipolar, and the antenna parameter $\eta_{\overline{\mathcal{F}}}$ can predict the enhancement of near-field optical chirality.

Finally, we show that the antenna parameters $\eta_{\overline{\mathcal{F}}}$ and $\sigma_{\bar{X}}$ enable broad spectral tunability of chiral optical fields. Figure 5 a plots experimental $\eta_{\overline{\mathcal{F}}}$ spectra for R-NRDs with varying $h$. In addition to the decrease of $\eta_{\overline{\mathcal{F}}}$ shown in Figure 4, we observe a spectral redshift with increasing $h$. The experimental wavelengths at which the extrema of $\eta_{\overline{\mathcal{F}}}$ occur, shown with respect to $h$ in Figure 5d, reveal a linear trend that qualitatively agrees with the simulated $\eta_{\overline{\mathcal{F}}}$ in Figure 5 b. Similar linear shifts have 

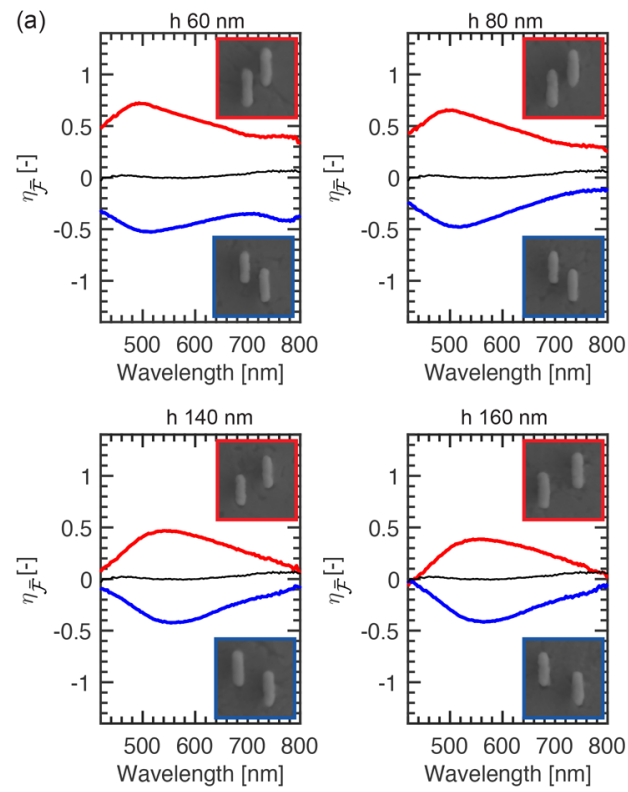
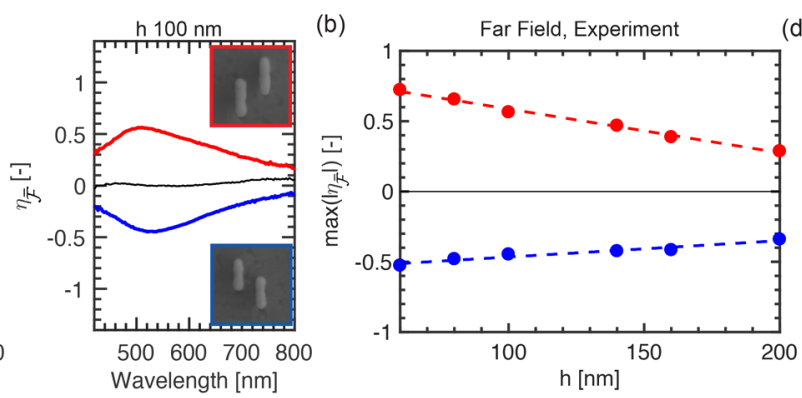

(c)

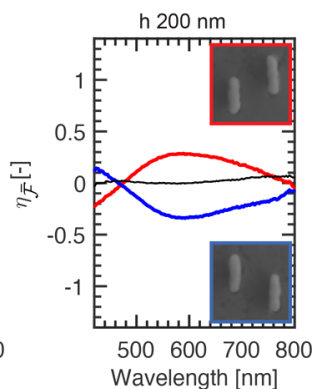

(d)
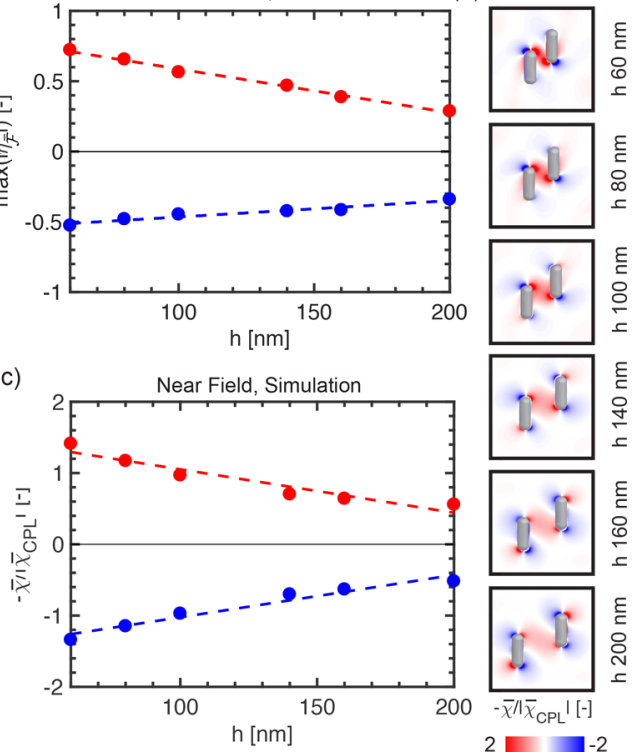

Figure 4. (a) Experimental chirality flux efficiency $\left(\eta_{\overline{\mathcal{F}}}\right)$ spectra for R-NRDs (red) and L-NRDs (blue) for varying horizontal shifts $h . \eta_{\overline{\mathcal{F}}}$ for achiral NRDs is shown at $h=60 \mathrm{~nm}$ (black). Insets: scanning-electron micrographs of a single NRD in the studied array. (b) Extrema of $\eta_{\overline{\mathcal{F}}}$ from part a. A linear trend with respect to $h$ (dashed lines provide linear fits) is observed. (c) Extrema of simulated $\bar{\chi} / \bar{\chi}_{\mathrm{CPL}} \mid$ evaluated at $(0,0,0)$ also show a linear trend with respect to $h$ (dashed lines provide linear fits). The data in parts $\mathrm{b}$ and $\mathrm{c}$ verify analytical calculations (eqs 9-11) and reveal that $h$ determines the magnetoelectric polarizability $\alpha_{\mathrm{c}}$ in this system. (d) Numerical colormaps of $\bar{\chi} / \bar{\chi}_{\mathrm{CPL}} \mathrm{I}(z=0$ plane) for R-NRDs further confirm the trends shown in part c.
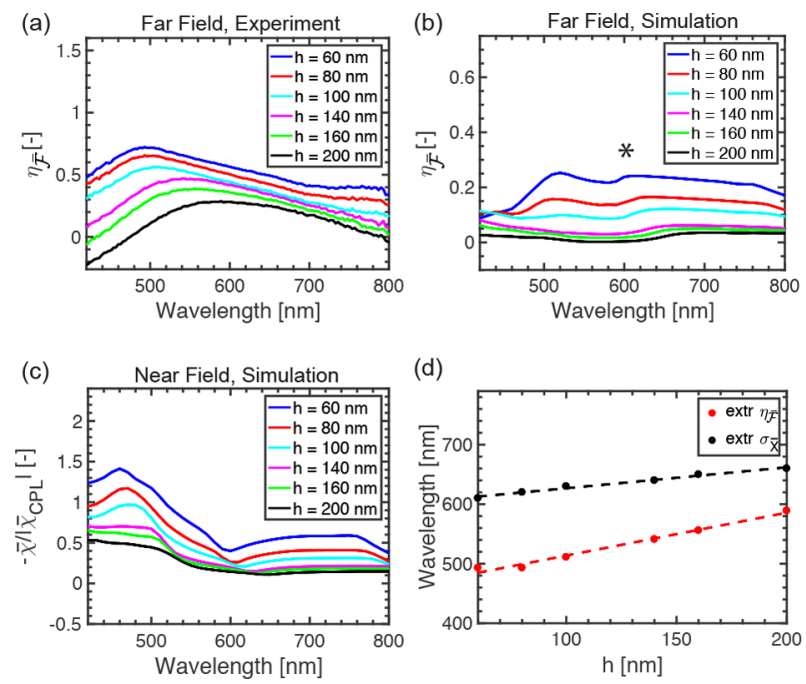

(d)

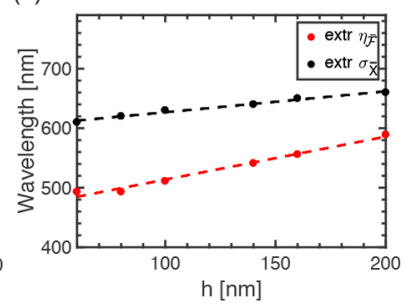

Figure 5. (a) R-NRD experimental chirality flux efficiency $\left(\eta_{\overline{\mathcal{F}}}\right)$ spectra for various horizontal shifts $h$. A redshift is observed with increasing $h$. (b) Corresponding simulated $\eta_{\overline{\mathcal{F}}}$ spectra are in agreement with experiments $(*$ indicates the surface lattice resonance). (c) Simulated $\bar{\chi} / \bar{\chi}_{\mathrm{CPL}} \mathrm{L}$ spectra evaluated at $(0,0,0)$ for R-NRDs replicate far-field trends seen in parts a and b. (d) Extrema of the dominant feature of experimental $\eta_{\overline{\mathcal{F}}}$ spectra and simulated chiral antenna aperture $\left(\sigma_{\bar{X}}\right)$ spectra for various $h$ (dashed lines provide linear fits). As $\sigma_{\bar{X}}$ is driven by the surface lattice resonance, the redshift is attributed to the increasing surface lattice distance. The results in part $\mathrm{d}$ reveal tunability of chiral fields across the visible spectrum.

been reported for energy and have been attributed to changes in the aspect ratio of the structure. ${ }^{56,68}$ As expected, comparable behavior is found in the chiral near fields (Figure 5c), evaluated at the R-NRD center $(0,0,0)$ (marked with $*$ in Figure $2 \mathrm{c}$ ).
The second antenna parameter $\sigma_{\bar{X}}$ (eq 4) provides further useful chiral optical information. Due to chirality conservation (Table 1 ), the integrated optical chirality dissipation (proportional to $\sigma_{\bar{X}}$ ) is equal to the optical chirality flux through the entire surface enclosing the studied structure. Thus, while we minimize the effect of surface lattice resonances (SLRs) by evaluating $\eta_{\overline{\mathcal{F}}}$ at small angles (SLRs are increasingly observed at higher angles, as shown in the Supporting Information), the dominant $\sigma_{\bar{X}}$ resonances are caused by SLRs in a $2 \mathrm{D}$ chiral system. As we designed our system with an edge-to-edge NRD distance $p$ of $600 \mathrm{~nm}$ (see Figure 2c), the effective surfacelattice-resonance wavelength increases with increasing $h$, as shown in Figure 5d. The spectral SLR redshift is also seen in the simulated $\eta_{\overline{\mathcal{F}}}$ spectra (marked with $*$ in Figure $5 \mathrm{~b}$ ), while damping and fabrication imperfections suppress this effect in experiment (Figure 5a). Indeed, this SLR redshift confirms our statement with regard to Figure $3 \mathrm{~b}$, where we attributed the resonance at $600 \mathrm{~nm}$ (marked with $*$ ) to a lattice effect (see the Supporting Information for further confirmation). The lowwavelength feature in Figure $5 b$, which arises with increasing $h$, can therefore be attributed to a higher-order lattice mode, explaining its absence in experiment. ${ }^{69}$ Thus, the tunability shown in Figure $5 \mathrm{~d}$ makes it apparent that our combined understanding of $\eta_{\overline{\mathcal{F}}}$ and $\sigma_{\bar{X}}$ enables the design of chiral optical fields across the visible spectrum.

In summary, we introduce the optical antenna approach to chiral plasmonics to fully characterize and tune antennamediated chiral light with the chiral antenna parameters $\eta_{\overline{\mathcal{F}}}$ and $\sigma_{\bar{X}}$. With chirality flux spectroscopy, a specifically designed experimental technique to determine $\eta_{\overline{\mathcal{F}}}$, we show that this approach provides valuable information on chiral near fields that is accessible in the far field. We verify this principle with simple $2 \mathrm{D}$ chiral coupled nanorod dimers and find that chiral 
near and far fields are linearly driven by the magnetoelectric polarizability. In analogy to energetic enhancement induced by classical optical antennas, this new approach can lead to optimized chiral optical applications, where highly chiral evanescent fields enhance the transmission of chiral information from a chiral receiver to the free radiation field.

\section{ASSOCIATED CONTENT}

\section{S Supporting Information}

The Supporting Information is available free of charge on the ACS Publications website at DOI: 10.1021/acs.nanolett.8b00083.

Experimental methods; derivations of plane- and evanescent-wave optical chirality density and dipole chiral antenna parameters; summary of the conservation law of optical chirality; scanning-electron micrograph images, extinction spectra, and CD spectra for all samples studied; discussion of experimental data analysis; characterization of achiral control samples; numerical methods; discussion of surface-lattice-resonance effects; simulated spectra of the chiral antenna aperture; and numerical geometry optimization (PDF)

\section{AUTHOR INFORMATION}

\section{Corresponding Author}

*E-mail: dnorris@ethz.ch.

\section{ORCID}

Lisa V. Poulikakos: 0000-0002-1118-789X

Eva De Leo: 0000-0002-9677-0274

David J. Norris: 0000-0002-3765-0678

\section{Author Contributions}

L.V.P. and D.J.N. conceived the project. L.V.P. developed the theory, performed analytical and numerical calculations, designed and built the optical setup, and analyzed the data. L.V.P., P.T., A.S., and E.D.L. carried out the sample fabrication. L.V.P., P.T., and A.S. performed the optical characterization. L.V.P. and D.J.N. wrote the manuscript with input from all authors.

\section{Funding}

This work was supported by the Swiss National Science Foundation under Award No. 200021-146747. D.J.N. acknowledges partial financial support from the European Research Council under the European Union's Seventh Framework Program (FP/2007-2013)/ERC Grant Agreement No. 339905 (QuaDoPS Advanced Grant).

\section{Notes}

The authors declare no competing financial interest.

\section{ACKNOWLEDGMENTS}

We thank H. Eghlidi, B. le Feber, F. Prins, A.-K. Michel, and N. Stricker for insightful discussions. We also thank P. Gutsche for helpful comments, in particular related to the optical chirality dissipation at interfaces. We thank S. A. Meyer for artistic rendering. We thank Y. Fedoryshyn and U. Drechsler for fabrication assistance.

\section{REFERENCES}

(1) Richardson, G. M. The Foundations of Stereo Chemistry: Memoirs by Pasteur, Van't Hoff, Lebel and Wislicenus; American Book Co.: New York, 1901.

(2) Hutt, A. J.; Tan, S. C. Drugs 1996, 52, 1-12.
(3) Rodger, A.; Nordén, B. Circular Dichroism and Linear Dichroism, 1st ed.; Oxford University Press: New York, 1997.

(4) Nakanishi, K.; Berova, N.; Woody, R. Circular Dichroism: Principles and Applications, 2nd ed.; Wiley-VCH: New York, 2000.

(5) Kelly, S. M.; Jess, T. J.; Price, N. C. Biochim. Biophys. Acta, Proteins Proteomics 2005, 1751, 119-139.

(6) Hentschel, M.; Schäferling, M.; Duan, X.; Giessen, H.; Liu, N. Sci. Adv. 2017, 3, e1602735.

(7) Schäferling, M.; Yin, X.; Giessen, H. Opt. Express 2012, 20, 26326-26336.

(8) García-Etxarri, A.; Dionne, J. A. Phys. Rev. B: Condens. Matter Mater. Phys. 2013, 87, 235409.

(9) Ho, C. S.; Garcia-Etxarri, A.; Zhao, Y.; Dionne, J. ACS Photonics 2017, 4, 197-203.

(10) Schäferling, M.; Dregely, D.; Hentschel, M.; Giessen, H. Phys. Rev. $X$ 2012, 2, 31010.

(11) Rotenberg, N.; Kuipers, L. Nat. Photonics 2014, 8, 919-926.

(12) Hendry, E.; Carpy, T.; Johnston, J.; Popland, M.; Mikhaylovskiy, R. V.; Lapthorn, A. J.; Kelly, S. M.; Barron, L. D.; Gadegaard, N.; Kadodwala, M. Nat. Nanotechnol. 2010, 5, 783-787.

(13) Tang, Y.; Cohen, A. E. Science 2011, 332, 333-336.

(14) Nesterov, M. L.; Yin, X.; Schäferling, M.; Giessen, H.; Weiss, T. ACS Photonics 2016, 3, 578-583.

(15) Canaguier-Durand, A.; Hutchison, J. A.; Genet, C.; Ebbesen, T. W. New J. Phys. 2013, 15, 123037.

(16) McPeak, K. M.; van Engers, C. D.; Bianchi, S.; Rossinelli, A.; Poulikakos, L. V.; Bernard, L.; Herrmann, S.; Kim, D. K.; Burger, S.; Blome, M.; Jayanti, S. V.; Norris, D. J. Adv. Mater. 2015, 27, 62446250.

(17) le Feber, B.; Rotenberg, N.; Kuipers, L. Nat. Commun. 2015, 6, 6695.

(18) Tullius, R.; Karimullah, A. S.; Rodier, M.; Fitzpatrick, B.; Gadegaard, N.; Barron, L. D.; Rotello, V. M.; Cooke, G.; Lapthorn, A.; Kadodwala, M. J. Am. Chem. Soc. 2015, 137, 8380-8383.

(19) Zhao, Y.; Saleh, A. A. E.; Dionne, J. A. ACS Photonics 2016, 3, 304-309.

(20) Zhao, Y.; Askarpour, A. N.; Sun, L.; Shi, J.; Li, X.; Alù, A. Nat. Commun. 2017, 8, 14180.

(21) Smith, K. W.; Link, S.; Chang, W. S. J. Photochem. Photobiol., C 2017, 32, 40-57.

(22) Poulikakos, L. V.; Gutsche, P.; McPeak, K. M.; Burger, S.; Niegemann, J.; Hafner, C.; Norris, D. J. ACS Photonics 2016, 3, 16191625.

(23) Bharadwaj, P.; Deutsch, B.; Novotny, L. Adv. Opt. Photonics 2009, 1, 438-483.

(24) Aćimović, S. S.; Kreuzer, M. P.; González, M. U.; Quidant, R. ACS Nano 2009, 3, 1231-1237.

(25) Novotny, L.; van Hulst, N. Nat. Photonics 2011, 5, 83-90.

(26) Tang, Y.; Cohen, A. E. Phys. Rev. Lett. 2010, 104, 163901.

(27) Joulain, K.; Carminati, R.; Mulet, J.-P.; Greffet, J.-J. Phys. Rev. B: Condens. Matter Mater. Phys. 2003, 68, 245405.

(28) Bliokh, K. Y.; Nori, F. Phys. Rev. A: At., Mol., Opt. Phys. 2011, 83, 21803.

(29) Novotny, L.; Hecht, B. Principles of Nano-Optics, 2nd ed.; Cambridge University Press: Cambridge, U.K., 2012.

(30) Schellman, J.; Jensen, H. P. Chem. Rev. 1987, 87, 1359-1399.

(31) Simpson, G. J. J. Chem. Phys. 2002, 117, 3398-3410.

(32) Bustamante, C.; Tinoco, I.; Maestre, M. F. Proc. Natl. Acad. Sci. U. S. A. 1983, 80, 3568-3572.

(33) Turner, D. H.; Tinoco, I.; Maestre, M. J. Am. Chem. Soc. 1974, 96, 4340-4342.

(34) Valev, V. K.; Silhanek, A. V.; Smisdom, N.; De Clercq, B.; Gillijns, W.; Aktsipetrov, O. A.; Ameloot, M.; Moshchalkov, V. V.; Verbiest, T. Opt. Express 2010, 18, 8286-8293.

(35) Valev, V. K.; Baumberg, J. J.; De Clercq, B.; Braz, N.; Zheng, X.; Osley, E. J.; Vandendriessche, S.; Hojeij, M.; Blejean, C.; Mertens, J.; Biris, C. G.; Volskiy, V.; Ameloot, M.; Ekinci, Y.; Vandenbosch, G. A. E.; Warburton, P. A.; Moshchalkov, V. V.; Panoiu, N. C.; Verbiest, T. Adv. Mater. 2014, 26, 4074-4081. 
(36) Rodrigues, S. P.; Lan, S.; Kang, L.; Cui, Y.; Cai, W. Adv. Mater. 2014, 26, 6157-6162.

(37) Rodrigues, S. P.; Cui, Y.; Lan, S.; Kang, L.; Cai, W. Adv. Mater. 2015, 27, 1124-1130.

(38) Lu, X.; Wu, J.; Zhu, Q.; Zhao, J.; Wang, Q.; Zhan, L.; Ni, W. Nanoscale 2014, 6, 14244-14253.

(39) Wang, L.-Y.; Smith, K. W.; Dominguez-Medina, S.; Moody, N.; Olson, J. M.; Zhang, H.; Chang, W.-S.; Kotov, N.; Link, S. ACS Photonics 2015, 2, 1602-1610.

(40) Smith, K. W.; Zhao, H.; Zhang, H.; Sánchez-Iglesias, A.; Grzelczak, M.; Wang, Y.; Chang, W. S.; Nordlander, P.; Liz-Marzán, L. M.; Link, S. ACS Nano 2016, 10, 6180-6188.

(41) Mark, A. G.; Gibbs, J. G.; Lee, T.-C.; Fischer, P. Nat. Mater. 2013, 12, 802-807.

(42) Cui, Y.; Kang, L.; Lan, S.; Rodrigues, S.; Cai, W. Nano Lett. 2014, 14, 1021-1025.

(43) Han, C.; Leung, H. M.; Chan, C. T.; Tam, W. Y. Opt. Express 2015, 23, 33065.

(44) Hu, L.; Tian, X.; Huang, Y.; Fang, L.; Fang, Y. Nanoscale 2016, $8,3720-3728$.

(45) Esposito, M.; Tasco, V.; Todisco, F.; Cuscunà, M.; Benedetti, A.; Scuderi, M.; Nicotra, G.; Passaseo, A. Nano Lett. 2016, 16, 58235828.

(46) Fernandez-Corbaton, I.; Fruhnert, M.; Rockstuhl, C. Phys. Rev. $X$ 2016, 6, 31013.

(47) Zhang, H.; Govorov, A. O. Phys. Rev. B: Condens. Matter Mater. Phys. 2013, 87, 75410.

(48) Riehl, J. P.; Richardson, F. S. Chem. Rev. 1986, 86, 1-16.

(49) Meinzer, N.; Hendry, E.; Barnes, W. L. Phys. Rev. B: Condens. Matter Mater. Phys. 2013, 88, 41407.

(50) Cotrufo, M.; Osorio, C. I.; Koenderink, A. F. ACS Nano 2016, 10, 3389-3397.

(51) Pompa, P. P.; Martiradonna, L.; Della Torre, A.; Della Sala, F.; Manna, L.; De Vittorio, M.; Calabi, F.; Cingolani, R.; Rinaldi, R. Nat. Nanotechnol. 2006, 1, 126-130.

(52) Gutsche, P; Poulikakos, L. V.; Hammerschmidt, M.; Burger, S.; Schmidt, F. Proc. SPIE 2016, 97560X.

(53) Sersic, I.; Tuambilangana, C.; Kampfrath, T.; Koenderink, A. F. Phys. Rev. B: Condens. Matter Mater. Phys. 2011, 83, 245102.

(54) Sersic, I.; van de Haar, M. A.; Arango, F. B.; Koenderink, A. F. Phys. Rev. Lett. 2012, 108, 223903.

(55) Barron, L. D. Molecular Light Scattering and Optical Activity, 2nd ed.; Cambridge University Press: Cambridge, U.K., 2009.

(56) Jain, P. K.; El-Sayed, M. A. Chem. Phys. Lett. 2010, 487, 153164.

(57) Yin, X.; Schäferling, M.; Metzger, B.; Giessen, H. Nano Lett. 2013, 13, 6238-6243.

(58) Nagpal, P.; Lindquist, N. C.; Oh, S.-H.; Norris, D. J. Science 2009, 325, 594-597.

(59) McPeak, K. M.; van Engers, C. D.; Blome, M.; Park, J. H.; Burger, S.; Gosálvez, M. A.; Faridi, A.; Ries, Y. R.; Sahu, A.; Norris, D. J. Nano Lett. 2014, 14, 2934-2940.

(60) Zhao, Y.; Belkin, M. A.; Alù, A. Nat. Commun. 2012, 3, 870.

(61) Yu, N.; Aieta, F.; Genevet, P.; Kats, M. A.; Gaburro, Z.; Capasso, F. Nano Lett. 2012, 12, 6328-6333.

(62) Plum, E.; Fedotov, V. A.; Zheludev, N. I. J. Opt. A: Pure Appl. Opt. 2009, 11, 074009.

(63) Tian, X.; Fang, Y.; Sun, M. Sci. Rep. 2015, 5, 17534.

(64) Zuloaga, J.; Nordlander, P. Nano Lett. 2011, 11, 1280-1283.

(65) Magkiriadou, S.; Park, J.-G.; Kim, Y.-S.; Manoharan, V. N. Phys. Rev. E 2014, 90, 062302.

(66) Jackson, J. D. Classical Electrodynamics, 3rd ed.; John Wiley \& Sons, Inc.: New York, NY, 1999.

(67) Nordlander, P.; Oubre, C.; Prodan, E.; Li, K.; Stockman, M. I. Nano Lett. 2004, 4, 899-903.

(68) Link, S.; Mohamed, M. B.; El-Sayed, M. A. J. Phys. Chem. B 1999, 103, 3073-3077.

(69) Wang, D.; Yang, A.; Hryn, A. J.; Schatz, G. C.; Odom, T. W. ACS Photonics 2015, 2, 1789-1794.

\section{NOTE ADDED AFTER ASAP PUBLICATION}

This paper originally published ASAP on March 23, 2018. Additional changes were made throughout the paper, including a revision to Figure 5 and the addition of reference 52. The revised version was published on May 2, 2018. 